Tropical Journal of Pharmaceutical Research October 2010; 9 (5): 431-439

(C) Pharmacotherapy Group

Faculty of Pharmacy, University of Benin

Benin City, 300001 Nigeria.

All rights reserved.

Research Article

Available online at http://www.tjpr.org

\title{
Angiotensin Converting Enzyme Insertion/Deletion Gene Polymorphism: An Observational Study among Diabetic Hypertensive Subjects in Malaysia
}

\author{
Jaime Jacqueline Jayapalan ${ }^{1}$, Sekaran Muniandy ${ }^{1 *}$ and Chan \\ Siew Pheng ${ }^{2}$ \\ ${ }^{1}$ Department of Molecular Medicine, ${ }^{2}$ Department of Medicine, Faculty of Medicine, University of Malaya, Kuala \\ Lumpur, Malaysia
}

\begin{abstract}
Purpose: This study investigated the influence of angiotensin-1 converting enzyme (ACE) insertiondeletion (ID) gene polymorphism on the treatment responses of type 2 diabetic subjects at varying stages of nephropathy to ACE inhibitors (ACEI) with regard to blood pressure (MAP) and renal response (GFR).

Methods: The pharmacological effect of ACE inhibition on mean arterial pressure (MAP) and glomerular filtration rate (GFR) were observed among a total of 62 subjects for a short-term duration of 15 months. MAP and GFR were calculated by standard mathematical formulae while the ACE ID genotype was determined using triple primer PCR. The general linear model repeated measures were applied to study the modulation of ACE inhibition on these parameters.

Results: ACE ID genotyping of the 62 subjects showed that 19 (30.6\%) subjects had the II genotype, while $35(56.4 \%)$ subjects showed ID genotype and 8 (12.9\%) subjects had the DD genotype. Significant mean MAP reduction $(p<0.05)$ and null mean GFR changes $(p>0.05)$ from baseline values were observed among the subjects following antihypertensive treatment. However, when stratified according to ACE genotypes, no significant mean MAP and GFR changes were observed between genotypes following antihypertensive treatment ( $p>0.05$ ).
\end{abstract}

Conclusion: ACE ID gene polymorphism does not determine the treatment efficacy of ACE inhibitors in the Malaysian population.

Keywords: ACE genotype, ACE inhibitor, Type 2 diabetes mellitus, Diabetic nephropathy 


\section{INTRODUCTION}

Many trial-proven strategies have been developed in preventing and delaying the progression of nephropathy in patients with diabetes. Maintenance of tight blood glucose and blood pressure controls, encouraging weight reduction and cessation of smoking, treating dyslipidaemia, restricting intake of protein diet, prescribing acute high or chronic low doses of erythropoietin and early identification and referral of patients with potential risk for developing end-stage renal failure are some of the diabetic nephropathy management strategies being practiced in primary health care settings for subjects at risk for developing diabetic nephropathy [1-2]. In addition, novel therapies including sulodexide, a glycosaminoglycan, pyridoxamine, an advanced glycation end product inhibitor, ruboxistaurin, a protein kinase $\mathrm{C}$ inhibitor and aliskiren, a renin inhibitor are promising in retarding disease progression of diabetic nephropathy both in animal models and small clinical studies [3].

Antihypertensive treatment (AHT), particularly the use of ACE inhibitors (ACEI), have been reported to show beneficial reno-protective effects in patients with diabetic and nondiabetic nephropathy [4]. ACEls execute hypotensive effects by decreasing the formation of a potent vasoconstrictor, angiotensin-II and by decreasing the degradation of the potent vasodilator, bradykinin while affecting glomerular haemodynamics by decreasing the glomerular capillary pressure. They also have an antiproteinuric effect superior to other AHTs [5].

The II, ID and DD genotypes resulting from the polymorphic site of the ACE gene have been shown to have low, intermediate and high plasma expression, respectively. With that, the II genotype has been thought to confer protective benefits while DD genotype, is believed to confer deleterious effects on various disease pathogenetic mechanism particularly, diabetic nephropathy [6]. In addition, characterization of the ACE ID gene polymorphism has also been suggested for decision making regarding antihypertensive treatment regimens [7]. Hence, we investigated the role of ACE ID gene polymorphism in Malaysian diabetic nephropathic subjects in determining the effect of ACE inhibitors on mean arterial pressure (MAP) and glomerular filtration rate (GFR) in a short-term observational retrospective study. Additionally, we have also assessed the concomitant use of additional antihypertensive drugs to ACEI on the changes of mean MAP and GFR over time in these subjects.

\section{METHODS}

\section{Patient selection}

Enrolment of subjects was conducted at the outpatient diabetes/nephrology clinic at the University of Malaya Medical Center (UMMC), Kuala Lumpur. Approval from the Medical Ethics Committee (UMMC) and informed consent from all recruited subjects were obtained. A total of 256 patients with varying stages of nephropathy were reviewed for the study. Following a thorough review of the medical records of these subjects, only a total of 62 subjects with type 2 diabetes mellitus (T2DM) and with a mean age ( \pm SD) of $60.5 \pm 8.4$ (male: $38.7 \%$; female: $61.2 \%$ ) were selected for this retrospective observational study. Among these, 37 subjects were also on one or two antihypertensive drugs in addition to ACEI. They had been on ACEls consecutively for over 15 months with complete medical records from 2001 to 2007. All the diabetic patients included were hypertensive (BP > $140 / 90 \mathrm{mmHg}$ ) and with varying degrees of kidney damage (ranging from stages I - III) [8] prior to ACEI treatment.

\section{Clinical characteristics}

Mean arterial pressure (MAP) was approximated by the equation, $\{2 / 3$ * diastolic blood pressure $(\mathrm{DBP})\}+\{1 / 3$ * systolic blood 
pressure (SBP)\}. The ratio of total haemoglobin $(\mathrm{Hb})$ to $\mathrm{HbA}_{1 \mathrm{c}}$ concentration expressed by glycosylated hemoglobin $\left(\mathrm{HbA}_{1 \mathrm{c}}\right)$ concentration in percentage, determined by cyanide-free colorimetric and latex agglutination method, respectively, was computed [9]. The glomerular filtration rate (GFR) was estimated by Cockcroft-Gault (C$\mathrm{G})$ equation [10] as in Eq 1.

GFR $\mathrm{ml} / \mathrm{min}=\{(140$ - age $) \times$ weight $(\mathrm{kg})\} /\{72 \times$ serum creatinine $(\mathrm{mg} / \mathrm{dl})\}$

\section{ACE ID genotyping}

Highly purified and concentrated genomic deoxyribonucleic acid (DNA) was isolated using the Wizard ${ }^{R}$ Genomic DNA purification kit (Promega, Madison) based on a series of lysis, precipitation and desalting procedures. The DNA was then subjected to triple primer polymerase chain reaction (PCR) amplification of the insertion/deletion (I/D) polymorphic site of the ACE gene as previously described [11]. Briefly, PCR was performed in a final volume of $25 \mu \mathrm{l}$ containing $10 \mathrm{mM}$ Tris- $\mathrm{HCl}(\mathrm{pH} 8.8), 50 \mathrm{mM} \mathrm{KCl}, 0.8 \%$ Nonidet P40, $1.5 \mathrm{mM} \mathrm{MgCl} 2,0.2 \mathrm{mM}$ dNTPs, 20pmol of each primer (Forward: 5'-CCC ATC CTT TCT CCC ATT TCT C-3', Nested: 5'-GGT TTC ACC GTT TTA GCC GGG A-3' and Reverse: 5'-CCA TGC CCA TAA CAG GTC TTC A-3' primers, $1 U$ Taq DNA Polymerase (Fermentas, Lithuania), and $\sim 100$ ng of genomic DNA. The optimized PCR run includes an initial denaturation at $94{ }^{\circ} \mathrm{C}$ for $3 \mathrm{~min}$, followed by 30 cycles of denaturation at $94{ }^{\circ} \mathrm{C}$ for $30 \mathrm{~s}$, annealing at $64{ }^{\circ} \mathrm{C}$ for $30 \mathrm{~s}$, and extension at $72{ }^{\circ} \mathrm{C}$ for 30 $\mathrm{s}$, and a final extension at $72{ }^{\circ} \mathrm{C}$ for $7 \mathrm{~min}$ in an Eppendorf Master Thermal Cycler (Eppendorf, Hamburg). Subsequently, following agarose gel (1.5\%) electrophoresis, ACE I/D genotyping was determined based on the discriminating band size pattern where band sizes of approximately 500 bp and 260 bp designated the II genotype, while band sizes of approximately $500 \mathrm{bp}, 260 \mathrm{bp}$ and 210 bp designated the ID genotype and a single band with a size of approximately 210 bp indicates the DD genotype.
ACE inhibitors (ACEI) observational
retrospective study

For the retrospective study, blood pressure readings were recorded from the subject's respective medical records at 3 monthly intervals. Subsequently, the MAP was estimated thus, showing a total of 6 readings for the period under study. C-G equationsbased GFR measures were estimated as well. Zero (0) month is the onset point of drug administration, and therefore, considered as the baseline value.

\section{Statistical analysis}

Statistical Package for Social Sciences version 14.0 (Chicago, Illinois) was used to perform statistical analysis. Paired samples Students t-test was used to compare the means of $\mathrm{HbA}_{1 \mathrm{c}}$ level between baseline and at month 15. Parametric one-way ANOVA procedure was used to test the mean MAP and GFR differences between the subgroups: (a) ACE genotype subgroups - II : ID : DD, and (b) Pharmacologic therapy subgroup ACEI : ACEI + one AHT : ACEI + two AHT, at baseline. Post-hoc analysis was carried out using the Tukey post-hoc test as equal variances were assumed. The general linear model (GLM) repeated measures were applied to study the effects of ACE inhibitors on MAP and GFR over the period. Mauchly's sphericity test was used to assess the assumption of sphericity, while the Bonferonni procedure was used to perform pairwise comparison between the groups of means. A $p$ value of $<0.05$ was considered statistically significant.

\section{RESULTS}

\section{ACE ID genotyping and clinical characteristics}

Typical gel presentation showing the isolated DNA and PCR amplicons banding pattern based ACE ID genotyping are shown in Figures 1 and 2, respectively. Clinical

Trop J Pharm Res, October 2010; 9 (5):433 


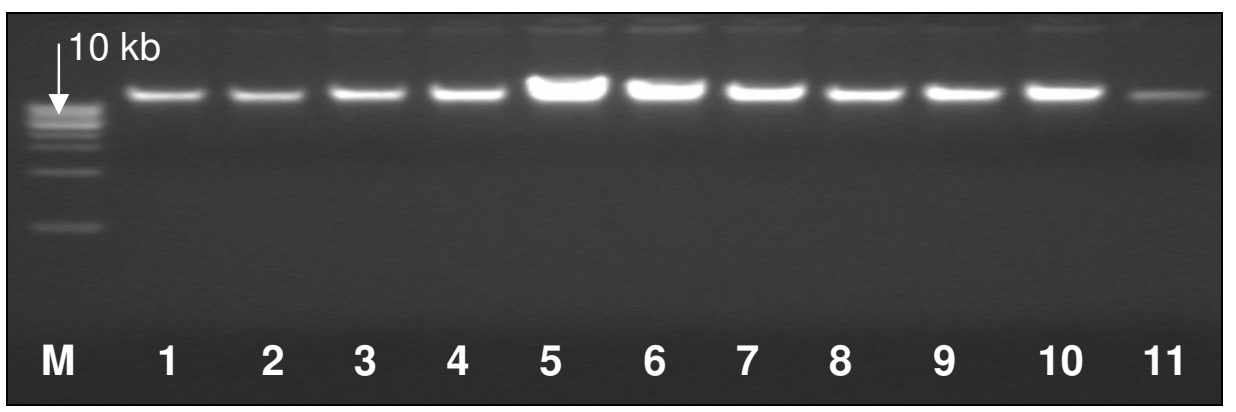

Figure 1: DNA isolates. (Lane M: 1Kb DNA ladder, lanes 1-11: DNA isolates)

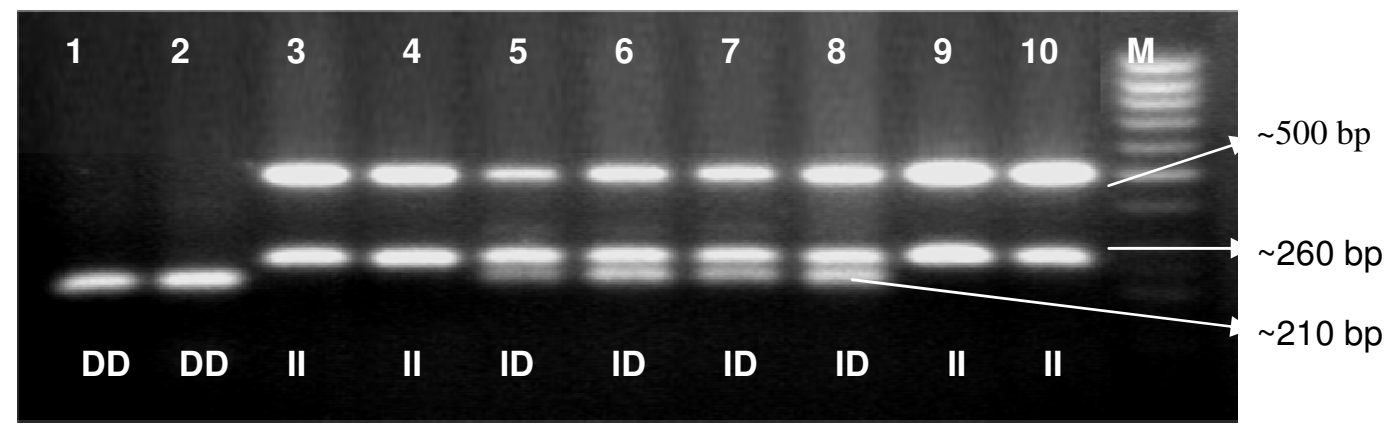

Figure 2: Typical ACE ID genotyping results by triple primer PCR. (Lane M: 50 bp DNA ladder; lanes 1-10: PCR product of samples in duplicate and its corresponding genotypes)

Table 1: Clinical characteristics (mean \pm SD) of subjects at baseline and endpoint of the study

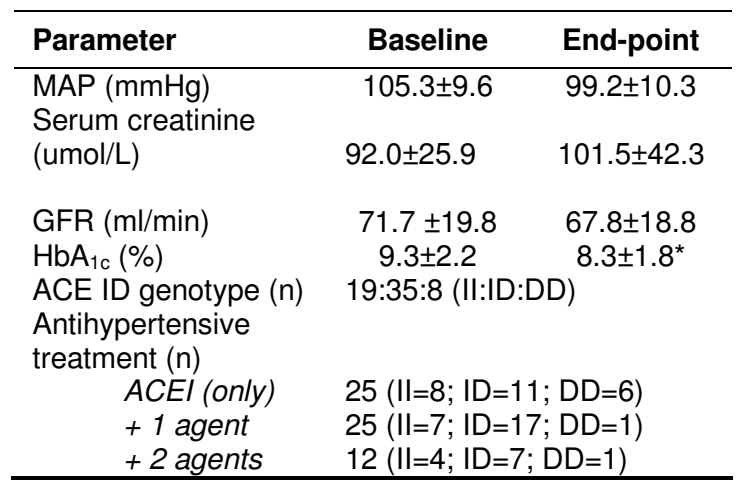

$M A P=$ mean arterial pressure; GFR = glomerular filtration rate; "Paired t-test, $\mathrm{HbA}_{1 c}$ levels at 15 months was significantly lower than the baseline value $(p<0.01)$.

characteristics of the subjects at both baseline and end-point are shown in Table 1. Table 1 indicates that homozygous insertion (II) alleles were found in 19 (30.6\%) patients while heterozygous insertion-deletion (ID) alleles and homozygous deletion alleles (DD) were found in $35(56.5 \%)$ and 8 patients $(12.9 \%)$, respectively. In terms of the antihypertensive therapy (AHT) regimens that had been administered to these subjects, $40.3 \%$ of the patients were on ACE inhibitors alone while $40.3 \%$ and $19.4 \%$ were on one and two additional antihypertensive agents, respectively (Table 1 ). The commonly used ACEl drugs include perindopril, lisinopril, enalapril and captopril, in that order. The types of drugs used in combination with ACE inhibitors were dihydropyridine calciumchannel blockers (CCBs) including amlodipine, nifedipine and felodipine, as well as diuretics - thiazide and furosemide.

\section{Responses of MAP and GFR to antihypertensive treatment}

The $p$ value for Mauchly test for all parameters tested was more than 0.05 , hence the assumption of sphericity was met $(p>0.05)$. As expected, test of within-

Trop J Pharm Res, October 2010; 9 (5):434 
Table 2: MAP and GFR responses to antihypertensive treatment over the duration of treatment

\begin{tabular}{lcccccc}
\hline $\begin{array}{l}\text { Clinical parameter } \\
\text { (mean } \pm \text { SEM) }\end{array}$ & $\mathbf{0}$ & $\mathbf{3}$ & $\mathbf{6}$ & $\mathbf{9}$ & $\mathbf{1 2}$ & $\mathbf{1 5}$ \\
\hline $\mathrm{MAP}(\mathrm{mmHg})$ & $104.7 \pm 1.5$ & $103.7 \pm 1.7$ & $105.2 \pm 1.5$ & $104.1 \pm 1.9$ & $100.9 \pm 1.7$ & $97.6 \pm 1.5$ \\
GFR $(\mathrm{ml} / \mathrm{min})$ & $69.8 \pm 3.5$ & $70.7 \pm 3.7$ & $71.3 \pm 3.4$ & $69.8 \pm 3.9$ & $70.4 \pm 4.0$ & $69.2 \pm 3.5$ \\
\hline
\end{tabular}

subjects effects showed a significant difference in the changes of mean MAP $(p=$ 0.001 , Observed power, $95.6 \%$ ) but not in mean GFR ( $p=0.963$ ) over 15 months (see Table 2). Using the Bonferonni pairwise comparison, we found that there was a significant difference in mean MAP measures between months 1, 3, 6 and 15 .

\section{Influence of combining other additional antihypertensive agents with ACEI on MAP and GFR}

Assessment of the influence of combining other AHTs with ACEI on mean MAP and GFR over time is shown in Figure 3. At baseline, the mean MAP of the subgroup on ACEI with 2 additional antihypertensive drugs was significantly higher compared to those on ACEI alone ( $p=0.03$; Tukey post hoc test, $p$ $<0.05)$. However, the mean baseline GFR between the subgroups ( $p=0.929)$ was not significantly different. Based on the test of between-subjects effects, it was observed that there was a significant mean MAP change between subgroups $(p<0.00$; Observed power, $99 \%$ ) but not in the mean GFR $(p=0.575)$ over time (Figure 3). Bonferonni pairwise comparisons showed a significantly lower mean MAP at 15 months from the baseline mean MAP among those on ACE inhibitors with two additional antihypertensive agents.

\section{Impact of ACE ID gene polymorphism on MAP and GFR and antihypertensive treatment outcomes}

Mean MAP and GFR were similar when stratified according to ACE ID genotypes at baseline ( $p=0.537$ and 0.571 , respectively). Further test of between-subjects effects between mean MAP and GFR and ACE ID genotypes gave $p$ values of 0.634 and 0.372 , respectively, indicating that there were no significant mean MAP and GFR changes between the subgroups over time (Figure 4).
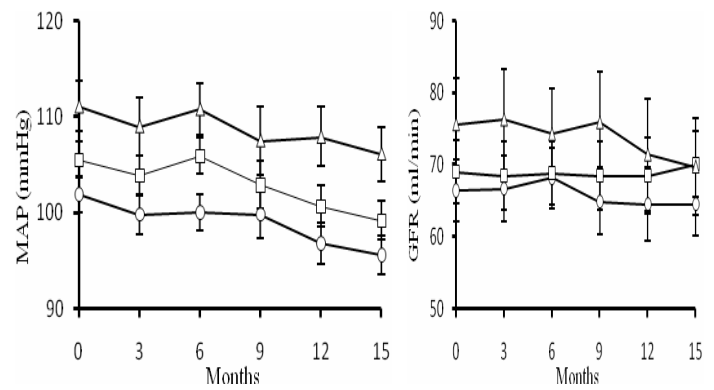

Figure 3: Mean MAP and GFR combined ACEI and other AHTs over the duration of treatment. (Key: $\circ$ = ACEI; $\square=\mathrm{ACEI}+$ one AHT; $\Delta=\mathrm{ACEI}+$ two AHT).
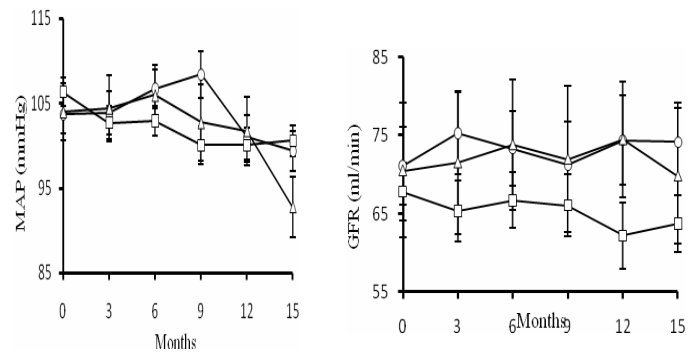

Figure 4: Mean MAP and GFR according to ACE genotype over the duration of treatment (Key: $\circ=$ II; $\square=$ ID; $\Delta=$ DD)

\section{Influence of ACE ID gene polymorphism on MAP and GFR for ACEI alone}

Changes in the mean MAP and GFR were also evaluated in subjects who were exclusively treated with ACEI $(n=25$; $I I=8$, $I D=11, D D=6)$. And again, no significant 
changes in both the MAP $(p>0.05)$ and GFR $(p>0.05)$ between subgroups were observed (data not shown).

\section{DISCUSSION}

The aim of this retrospective study was to observe the influence of ACE ID gene polymorphism on the responses of MAP and GFR to ACE inhibitors over period of 15 months. The effects of other antihypertensive agents administered concurrently with ACEI on changes of MAP and GFR over this period were also assessed.

The study revealed that (a) ACEI exerted the expected hypotensive effect and maintained renal function (GFR) among the T2DM subjects with variable baseline degrees of renal insufficiency for that duration of time, and (b) ACE ID gene polymorphism had no demonstrable influence on the antihypertensive effects or the maintenance of kidney function among these subjects.

\section{MAP}

Overall, the use of antihypertensive agents (ACE inhibitor alone or with other second-line agents) significantly decreased the MAP from baseline values. ACEls had been used as first line therapy among these subjects as recommended [12]. Only in uncontrolled BP situations, second-line agents were added. In the present study, aggressive use of additional antihypertensive agents were only prescribed for patients with MAP $110 \mathrm{mmHg}$ (SBP/DBP, 150/90). In such subjects, the dihydropyridine CCBs and diuretics were the only types of drugs used as second-line antihypertensive agents. These agents are well documented for exerting hypotensive effects, especially when given in combination with ACEI [13]. Similarly in the present study, a higher magnitude of change in mean MAP was observed among those on ACEI with two additional antihypertensive agents indicating synergistic drug interaction.

The observed erratic change in MAP averages among subjects between clinic visits is presumably attributable to poor compliance to treatment. Non-compliance including failure to replenish prescription, consumption of incorrect dose, irregular timing, forgetfulness, and premature discontinuation of drugs would generally prove to be significant impediments to effective treatment [14].

\section{GFR}

Cockcroft-Gault equations were used to estimate GFR which, in turn, was used as a marker to assess renal function of the subjects. Alternatively, the Modified Diet in Renal Disease (MDRD) equation may also be used for this purpose [15]. Although neither serves as an adequate GFR predicting formula, cost effectiveness and test convenience renders them acceptable for short-term studies [16]. Over the 15-month duration of the study, GFR was maintained at baseline values. The natural course of diabetic nephropathy has an average decline in GFR of $10-15 \mathrm{ml} / \mathrm{min} /$ year [1]. Hence, AHT regimens including ACEI as monotherapy or in combination with dihydropyridine CCBs and diuretics, have served their purpose by maintaining kidney function and preventing further decline in GFR.

As this is a retrospective study, it is rather difficult to determine the class of antihypertensive agents that contributed most to GFR maintenance especially when GFR trend were similar in all the subgroups compared (ACEl vs ACEl+one AHT vs ACEl+two AHTs), irrespective of the class/number of antihypertensive agents used. A critical appraisal of the data suggests that there was no significant synergistic antiproteinuric effect of dihydropyridine CCBs and diuretic type of antihypertensive drugs in the present study, unlike other studies that reported beneficial renoprotective effect when other drugs such as angiotensin II receptor antagonist [17] and potassium-sparring diuretics [18] were used in combination with ACEls. This implies that ACEI, a common 
denominator in all the subgroups may have been the major contributor to GFR maintenance. Although the protective influence of ACE inhibition against deterioration in renal function is well known as it exerts both anti-hypertensive as well as anti-proteinuric effects [4,5], the GFR maintenance observed in the present study may also have been influenced by improved blood pressure and glycaemic control among the subjects, as $\mathrm{HbA}_{1 \mathrm{C}}$ values at month 15 were significantly lower than at baseline. A prospective observational study had previously associated incidences of diabetes complications with glycaemic exposure. Reduction in haemoglobin $A_{1 c}$ by $1 \%$ correlated with a decrease in risk for microvascular complications and end-point or death related to diabetes by 37 and $21 \%$, respectively [19]. Therefore, further validation based on a long-term (observational) study involving additional judiciously selected subgroups (such as a non-ACEl subgroup) may be necessary to confirm this assertion.

\section{ACE ID gene polymorphism}

Therapeutic outcomes of ACE inhibition were previously attributed to polymorphism of the ACE gene. DD genotype is associated with high-level availability of Ang-II and lower availability level of bradykinin in the kidneys, causing higher efferent arteriolar resistance and intraglomerular pressure while patients with ID and II genotype have an intermediate and decreased level of vascular and renal activity, respectively [6]. Hence, ACE inhibition was associated with improved survival and renoprotection, with the beneficial effects more pronounced in subjects with the II and ID genotypes [7]. In contrast, Scharplatz et al [20] showed a better response to ACE inhibitors in Caucasian DD genotype carriers compared to II genotype carriers in terms of blood pressure, proteinuria, GFR, ACE activity and progression to ESRF in a preliminary systematic review.
In the present study however, there was no apparent impact of ACE ID gene polymorphism on the outcomes of antihypertensive therapy with regards to MAP and GFR in the subjects with diabetic nephropathy. The influence of ACE ID gene polymorphisms on the treatment outcome with ACE inhibitors have previously been demonstrated in subjects with type 1 diabetic nephropathy [21] and type 2 diabetic nephropathy $[6,7]$ and non-diabetic nephropathies [22]. Although our finding is in contrast to these studies, but it is in agreement with reports by van der Kleij et al [23] and Schelleman et al [24] in non-diabetic renal disease and hypertensive subjects, respectively. The authors reported that the decline in GFR and blood pressure, in the latter study, in response to ACE inhibition was not affected by ACE genotype.

\section{Limitations of this study}

The observed lack of association between genotype and response to ACE inhibition in the present study could be due to the variation in the pharmacokinetics and pharmacodynamics of the type of ACE inhibitors [25] prescribed for the subjects. Furthermore, the small sample size involved in the study made it impossible to assess the effects of genotype for the different ACE inhibitors separately. In addition, short duration of follow-up period [23], as well as heterogeneity in ethnicity [26] and gender [27] may also have hampered a significant association of ACE genotypes. Further studies exclusively designed to accommodate these pharmacologic and demographic factors is deemed necessary to confirm our present finding of null influence of ACE ID gene polymorphism on response to ACE inhibitors.

\section{CONCLUSION}

In this study on Malaysian diabetic subjects, ACE ID gene polymorphism had little impact on the efficacy of ACE inhibitor therapy with regard to MAP and GFR. 


\section{ACKNOWLEDGEMENT}

This work was funded by UM-PPP Grant P0103/2006B and PS290/2007B. Also, the authors wish to express their deep gratitude to Assoc Prof. Dr Karuthan Chinna of UITM, Malaysia, who assisted with the statistics used in this work.

\section{REFERENCES}

1. American Diabetes Association. Nephropathy in diabetes. Diabetes Care 2004; 27(1): S79S83.

2. Fliser $D$, Bahlmann $F H$, Haller $H$. EPO: Renoprotection beyond anemia correction. Pediatr Nephrol 2006; 21(12): 1785-1789.

3. Burney BO, Kalaitzidis RG, Bakris GL. Novel therapies of diabetic nephropathy. Curr Opin Nephrol Hypertens 2009; 18(2): 107-111.

4. Kshirsagar AV, Joy MS, Hogan SL, Falk RJ, Colindres RE. Effect of ACE inhibitors in diabetic and non-diabetic chronic renal disease: A systematic overview of randomized placebo-controlled trials. Am J Kidney Dis 2000; 35(4): 695-707.

5. Strippoli GF, Craig M, Craig JC. Antihypertensive agents for preventing diabetic kidney disease. Cochrane Database Syst Rev 2005; 19(4): CD004136.

6. Jacobsen PK. Preventing end stage renal disease in diabetic patients: Genetic aspect (part 1). JRAAS 2005; 6(1): 1-14.

7. So $W Y$, Ma RC, Ozaki R, Tong PC, Ng MC, Ho CS, Lam CW, Chow CC, Chan WB, Kong AP, et al. Angiotensin-converting enzyme (ACE) inhibition in type 2 diabetic patients: Interaction with ACE insertion/deletion polymorphism. Kidney Int 2006; 69(8): 1438-1443.

8. National Kidney Foundation-Kidney Disease Outcomes Quality Initiative (NKF K/DOQI). Clinical practice guidelines for cardiovascular disease in dialysis patients. New York: National Kidney Foundation; 2005.

9. Jeppsson JO, Kobold U, Finke A, Hoelzel W, Hoshino T, Miedema K, Mosca A, Mauri $P$, Paroni R, Thienpont $L$, et al. Approved IFCC reference method for the measurement of $\mathrm{HbA}_{1 \mathrm{C}}$ in human blood. Clin Chem Lab Med 2002; 40(1): 78-89.

10. Cockcroft DW, Gault MH. Prediction of creatinine clearance from serum creatinine. Nephron 1976; 16(1): 31-41.

11. Jayapalan JJ, Muniandy S, Chan SP. Angiotensin-1 converting enzyme I/D gene polymorphism: Scenario in Malaysia. Southeast Asian J Trop Med Public Health 2008; 39(5): 917-921.
12. American Diabetes Association. Position statement: Standards of medical care in diabetes-2007. Diabetes Care 2007; 30(1): S1-S41.

13. Ruggenenti $P$, Fassi $A$, llieva AP, Bruno $S$, lliev IP, Brusegan V, Rubis N, Gherardi G, Arnoldi F, Ganeva $M$, et al. Preventing microalbuminuria in type 2 diabetes. N Engl $J$ Med 2004; 351(19): 1941-1951.

14. Majtás J, Milly M, Slizová M, Failová S, Molnárová E. Therapeutic compliance and its pharmaceutical aspects. Ceska Slov Farm 2000; 49(5): 229-234.

15. Levey AS, Coresh J, Balk E, Kausz AT, Levin A, Steffers MW, Hogg RJ, Peronne RD, Lau J, Eknoyan G. National Kidney Foundation practice guidelines for chronic kidney disease: Evaluation, Classification, and Stratification. Ann Intern Med 2003; 139(2): 137-147.

16. Rigalleau V, Lasseur C, Perlemoine C, Barthe $N$, Raffaitin $C$, Chung $L$, Chauveau $P$, BailletBlanco L, Beauvieux MC, Combe $C$, et al. Estimation of glomerular filtration rate in diabetic subjects. Cockcroft formula or modification of diet in renal disease study equation? Diabetes Care 2005; 28(4): 838843.

17. Strippoli GF, Bonifati C, Craig M, Navaneethan $S D$, Craig JC. Angiotensin converting enzyme inhibitors and angiotensin II receptor antagonists for preventing the progression of diabetic kidney disease. Cochrane Database Syst Rev 2006; 18(4): CD006257.

18. Ustundag $A$, Tugrul $A$, Ustundag $S$, Sut $N$, Demirkan $B$. The effects of spironolactone on nephron function in patients with diabetic nephropathy. Ren Fail 2008; 30(10): 982-991.

19. UK Prospective Diabetes Study (UKPDS) 35. Association of glycaemia with macrovascular and microvascular complications of type 2 diabetes: Prospective observational study. BMJ 2000; 321(7258): 405-412.

20. Scharplatz M, Puhan MA, Steurer J, Perna A, Bachmann LM. Does the angiotensinconverting enzyme (ACE) gene insertion/deletion polymorphism modify the response to ACE inhibitor therapy? Curr Control Trials Cardiovasc Med 2005; 6(1): 16.

21. Tarnow $L$, Parving $H H$, Jacobsen $P$, Rossing $P$, Lecerf L, Porier $O$, Cambien $F$. The significance of deletion polymorphism in the ACE gene for progression of diabetic nephropathies treated with ACE inhibitors. Ugeskr Laeger 1998; 160(34): 4886-4889.

22. van Essen GG, Rensma PL, de Zeeuw D, Sluiter WJ, Scheffer $H$, Apperloo AJ, de Jong PE. Association between angiotensin converting enzyme gene polymorphism and failure of reno-protective therapy. Lancet 1996; 347(8994): 94-95.

23. van der Kleij FG, Navis GJ, Gansevoort RT, Heeg $J E$, Scheffer $H$, de Zeeuw D, de Jong PE. ACE polymorphism does not determine short-term renal response to ACE-inhibition in proteinuric 
patients. Nephrol Dial Transplant 1997; 12(2): 42-46.

24. Schelleman $H$, Klungel $O H$, van Duijn $C M$, Witteman JC, Hofman A, de Boer A, Stricker $B H$. Drug-gene interaction between the insertion/deletion polymorphism of the angiotensin-converting enzyme gene and antihypertensive therapy. Ann Pharmacother 2006; 40(2): 212-218.

25. Apperloo AJ, de Zeeuw D, de Jong PE. Discordant effects of enalapril and lisinopril on systemic and renal hemodynamics. Clin Pharmacol Ther 1994; 56(6): 647-658.
26. Ding PY, Hu OY, Pool PE, Liao W. Does Chinese ethnicity affect the pharmacokinetics and pharmacodynamics of angiotensin-converting enzyme inhibitors? J Hum Hypertens 2000; 14(3): 163-170.

27. Ruggenenti $P$, Perna A, Zocalli C, Gherardi G, Benini $R$, Testa $A$, et al. Chronic proteinuric nephropathies, II. Outcomes and response to treatment in a prospective cohort of 352 patients: Differences between women and men in relation to the ACE gene polymorphism. Gruppo Italiano di Studi Epidemologici in Nefrologia (Gisen). J Am Soc Nephrol 2000; 11(1): 89-96. 